ELECTRONIC RESEARCH ANNOUNCEMENTS OF THE AMERICAN MATHEMATICAL SOCIETY

Volume 9, Pages 26-31 (February 14, 2003)

S $1079-6762(03) 00106-9$

\title{
MARKOV STRUCTURES FOR NON-UNIFORMLY EXPANDING MAPS ON COMPACT MANIFOLDS IN ARBITRARY DIMENSION
}

\author{
JOSÉ F. ALVES, STEFANO LUZZATTO, AND VILTON PINHEIRO
}

(Communicated by Svetlana Katok)

\begin{abstract}
We consider non-uniformly expanding maps on compact Riemannian manifolds of arbitrary dimension, possibly having discontinuities and/or critical sets, and show that under some general conditions they admit an induced Markov tower structure for which the decay of the tail of the return time function can be controlled in terms of the time generic points needed to achieve some uniform expanding behavior. As a consequence we obtain some rates for the decay of correlations of those maps and conditions for the validity of the Central Limit Theorem.
\end{abstract}

\section{Dynamical and geOmetrical assumptions}

Let $M$ be a compact Riemannian manifold of dimension $d \geq 1$ with a normalized Riemannian volume $|\cdot|$, which we call Lebesgue measure. Let $f: M \rightarrow M$ be a $C^{2}$ local diffeomorphism for all $x \in M \backslash \mathcal{C}$, where $\mathcal{C}$ is some critical set, which may include points at which the derivative $D f_{x}$ is degenerate, as well as points of discontinuity and points at which the derivative is infinite. We assume the following natural non-degeneracy condition on $\mathcal{C}$, which generalizes the notion of non-flat critical points for smooth one-dimensional maps.

Definition 1. The critical set $\mathcal{C} \subset M$ is non-degenerate if $|\mathcal{C}|=0$ and there is a constant $\beta>0$ such that for every $x \in M \backslash \mathcal{C}$ we have $\operatorname{dist}(x, \mathcal{C})^{\beta} \lesssim\left\|D f_{x} v\right\| /\|v\| \lesssim$ $\operatorname{dist}(x, \mathcal{C})^{-\beta}$ for all $v \in T_{x} M$, and the functions $\log \operatorname{det} D f$ and $\log \left\|D f^{-1}\right\|$ are locally Lipschitz with Lipschitz constant $\lesssim \operatorname{dist}(x, \mathcal{C})^{-\beta}$.

We now state our two dynamical assumptions: the first is on the growth of the derivative and the second is on the approach rate of orbit to the critical set. Notice that for a linear map $A$, the condition $\|A\|>1$ only provides information about the existence of some expanded direction, whereas the condition $\left\|A^{-1}\right\|<1$ (i.e., $\log \left\|A^{-1}\right\|^{-1}>0$ ) implies that every direction is expanded.

Received by the editors November 5, 2002.

2000 Mathematics Subject Classification. Primary 37D20, 37D50, 37C40.

Work carried out at the Federal University of Bahia, University of Porto and Imperial College, London. Partially supported by CMUP, PRODYN, SAPIENS and UFBA. 
Definition 2. We say that $f$ is non-uniformly expanding if there exists $\lambda>0$ such that

$$
\liminf _{n \rightarrow \infty} \frac{1}{n} \sum_{i=0}^{n-1} \log \left\|D f_{f^{i}(x)}^{-1}\right\|^{-1} \geq \lambda
$$

for almost every $x \in M$.

Definition 3. We say that $f$ satisfies the property of subexponential recurrence to the critical set if for any $\epsilon>0$ there exists $\delta>0$ such that for Lebesgue almost every $x \in M$

$$
\limsup _{n \rightarrow+\infty} \frac{1}{n} \sum_{j=0}^{n-1}-\log \operatorname{dist}_{\delta}\left(f^{j}(x), \mathcal{C}\right) \leq \epsilon .
$$

Here $d_{\delta}(x, \mathcal{C})$ denote the $\delta$-truncated distance from $x$ to $\mathcal{C}$ defined as $d_{\delta}(x, \mathcal{C})=$ $d(x, \mathcal{C})$ if $d(x, \mathcal{C}) \leq \delta$, and $d_{\delta}(x, \mathcal{C})=1$ otherwise.

For the proofs of some technical lemmas (in particular, Lemma 4) we need to fix $\varepsilon$ satisfying certain conditions, and some of the definitions below (in particular, Definition (4) depend on this choice. We suppose therefore that some suitable $\varepsilon$ and the corresponding $\delta$ are fixed for the rest of the paper.

Remark 1. It was proved in 2] that conditions $(*)$ and $(* *)$ imply the existence of an absolutely continuous invariant probability measure $\mu$ on $M$. Once such a measure is given, both conditions admit very natural equivalent formulations

$$
(*) \Leftrightarrow \int \log \left\|D f_{x}^{-1}\right\|^{-1} d \mu>0
$$

and

$$
(* *) \Leftrightarrow \int|\log \operatorname{dist}(x, \mathcal{C})| d \mu<\infty
$$

\section{MEASURING THE NON-UNIFORMITY}

The asymptotic, non-uniform, nature of conditions $(*),(* *)$ is one of the main reasons for the difficulties in studying the finer geometric structures and dynamical properties of $f$. To gain some control over this non-uniformity we introduce the following

Definition 4. Let $\Gamma_{n}=\{x: \mathcal{E}(x)>n$ or $\mathcal{R}(x)>n\}$, where

$$
\mathcal{E}(x)=\min \left\{N \geq 1: \frac{1}{n} \sum_{i=0}^{n-1} \log \left\|D f_{f^{i}(x)}^{-1}\right\|^{-1} \geq \lambda / 2 \quad \forall n \geq N\right\}
$$

is the expansion time function, and

$$
\mathcal{R}(x)=\min \left\{N \geq 1: \frac{1}{n} \sum_{i=0}^{n-1}-\log \operatorname{dist}_{\delta}\left(f^{j}(x), \mathcal{C}\right) \leq 2 \varepsilon, \forall n \geq N\right\}
$$

is the recurrence time function.

We think of $\mathcal{E}(x), \mathcal{R}(x)$ as the waiting times before the asymptotic behaviour kicks in. By $(*)$ and $(* *), \mathcal{E}$ and $\mathcal{R}$ are defined and finite a.e., and therefore $\left|\Gamma_{n}\right| \rightarrow 0$ as $n \rightarrow \infty$. The rate at which $\left|\Gamma_{n}\right|$ decays is, in some sense, a measure of the non-uniformity of $f$. Our main result shows that this intuition is reflected in certain geometrical properties of the dynamics of $f$. 


\section{Generalized Markov Partitions}

Before stating our main theorem we introduce the geometric structures in which we are interested.

Definition 5. We say that $f$ admits a Markov Tower or Generalized Markov Partition if there exists a ball $\Delta \subset M$, a countable partition $\mathcal{P}(\bmod 0)$ of $\Delta$ into topological balls $U$ with smooth boundaries, and a return time function $R: \Delta \rightarrow \mathbb{N}$ piecewise constant on elements of $\mathcal{P}$, satisfying the following properties:

1. Markov: for each $U \in \mathcal{P}$ and $R=R(U), f^{R}: U \rightarrow \Delta$ is a $C^{2}$ diffeomorphism. We let $F(x)=f^{R(x)}(x)$.

2. Uniform expansivity: $\exists \hat{\lambda}>1$ such that $\left\|D F(x)^{-1}\right\|^{-1} \geq \hat{\lambda}$ for a.e. $x \in \Delta$. In particular, the separation time $s(x, y)=\max \left\{k: F^{i}(x), F^{i}(y)\right.$ belong to the same element of $\mathcal{P}, \forall i \leq k\}$ is finite for a.e. pair $x, y$.

3. Bounded volume distortion: $\exists K>0$ such that $\left|\frac{\operatorname{det} D F(x)}{\operatorname{det} D F(y)}-1\right| \leq$ $K \hat{\lambda}^{-s(F(x), F(y))} \forall x, y$ with $s(x, y) \in[1, \infty)$.

The main difference between this and a standard Markov partition is that here the partition is not defined on the whole manifold $M$ but only on some possibly small subset $\Delta$, and that the Markov property is not verified after a single iterate of $f$ but after a variable, unbounded, number of iterates which depend on the partition element. These weaker conditions make it possible to prove the existence of Generalized Markov Partition in much more general situations than those for which standard Markov partitions exist.

\section{Statement of Results}

Theorem 1. Let $f: M \rightarrow M$ be a transitive $C^{2}$ local diffeomorphism outside a non-degenerate critical set $\mathcal{C}$ satisfying conditions $(*)$ and $(* *)$, and suppose that there exists $\gamma>0$ such that

$$
\left|\Gamma_{n}\right|=\mathcal{O}\left(n^{-\gamma}\right)
$$

Then $f$ admits a Generalized Markov Partition, and the return time function satisfies

$$
|\{x: R(x)>n\}|=\mathcal{O}\left(n^{-\gamma}\right) .
$$

There are several possible motivations for the construction of Generalized Markov Partitions; we refer to [3, 4] for a detailed discussion and references. We mention here one implication for statistical properties of the maps, which follows from our result and from [7, 8.

Corollary 1. Let $f: M \rightarrow M$ be a transitive $C^{2}$ local diffeomorphism outside a non-degenerate critical set $\mathcal{C}$ satisfying conditions $(*)$ and $(* *)$, and suppose that there exists $\gamma>0$ such that $\left|\Gamma_{n}\right|=\mathcal{O}\left(n^{-\gamma}\right)$. Then there exists an absolutely continuous, $f$-invariant, probability measure $\mu$. Some finite power of $f$ is mixing with respect to $\mu$, and for any Hölder continuous functions $\varphi, \psi$ on $M$ we have

$$
\mathcal{C}_{n}=\left|\int\left(\varphi \circ f^{n}\right) \psi d \mu-\int \varphi d \mu \int \psi d \mu\right|=\mathcal{O}\left(n^{-\gamma+1}\right) .
$$

Moreover, if $\gamma>2$, then the Central Limit Theorem holds.

In particular, we obtain the following results for the two-dimensional non-uniformly expanding Viana maps [6, [1]. 
Corollary 2. The Viana maps satisfy the Central Limit Theorem and exhibit super-polynomial decay of correlations, i.e.,

$$
\mathcal{C}_{n}=\mathcal{O}\left(n^{-\gamma}\right) \quad \forall \gamma>0 .
$$

\section{BASIC STRATEGY}

We restrict ourselves here to the outline of the main steps of the proof of the theorem; the details will appear in 3 and 4 . We observe first of all that the transitivity assumption implies the existence of a point $p$ with dense preimages, and choose some sufficiently small ball $\Delta_{0}$ centred at $p$. This will be the domain of definition of our induced map. The idea is to consider iterates of $\Delta_{0}$ until we find some $n_{0}$ such that $f^{n_{0}}\left(\Delta_{0}\right)$ completely covers $\Delta_{0}$ and some bounded distortion property is satisfied. Then there is $U \subset \Delta_{0}$ such that $f^{n_{0}}(U)=\Delta_{0}$, and $U$ is by definition an element of the final partition $\mathcal{P}$ with associated return time $R=n_{0}$. We then continue iterating the complement $\Delta_{0} \backslash U$ until more good returns occur. By taking some care in the construction, this does indeed yield a Generalized Markov Partition with the required bounds on the tail of the return times. For this purpose we need some more concrete geometrical and combinatorial information regarding the time it takes for given domains to grow in size and eventually cover $\Delta_{0}$, and on the geometry of the complement $\Delta_{0} \backslash U$. Indeed, iterating the construction, at time $n$ we will be dealing with the complement of an increasing number of domains corresponding to regions which have had good returns up to time $n$.

\section{REturning to A GIVEN DOMAIN}

Our first observation implies that it is sufficient for a domain to grow large enough with bounded distortion, to guarantee that a good return to $\Delta_{0}$ will then occur within some fixed maximum number of iterates.

Lemma 2. $\forall \delta>0, \exists N_{0} \in \mathbb{N}$ such that $\bigcup_{j=0}^{N_{0}} f^{-j}(\{p\})$ is $\delta$-dense in $M$ and disjoint from $\mathcal{C}$.

Thus any sufficiently large ball will contain a preimage of $p$ close to its centre, and the statement made above holds true. In paricular, it is sufficient to concentrate on the rate at which small regions grow to some fixed large scale.

\section{Growing to LARGe SCALE}

We approach this problem through the notion of hyperbolic times introduced in [1. We say that for a given $\delta_{1}>0, k$ is a hyperbolic time for $x$ if there exists a neighbourhood $V_{n}$ of $x$, called a hyperbolic preball, such that $f^{n}\left(V_{n}\right)$ is a ball of radius $\delta_{1}$ and the volume distortion of $f^{n}$ on $V_{n}$ is uniformly bounded by a given constant independent of $n$ or $x$. In particular, if $\delta \ll \delta_{1}$, using Lemma 2, it is possible to prove

Lemma 3. $\exists c>0$ such that if $n$ is a hyperbolic time for $x, \exists$ a neighbourhood $U \subset V_{n}$ of $x$ with $|U| /\left|V_{n}\right| \geq c$ such that $f^{n+i}(U)=\Delta_{0}$ for some $i \leq N_{0}$, and $f^{n+i}$ has bounded volume distortion on $U$.

Thus, the return time is controlled locally by the occurrence of a hyperbolic time. This is naturally related to the expansion and recurrence time functions through the following 
Lemma 4. $\exists \theta>0$ such that $\forall x$ and $n \geq \max \{\mathcal{E}(x), \mathcal{R}(x)\} \exists \theta n$ hyperbolic times $n_{1}<\cdots<n_{\theta n}<n$.

\section{Global Rate of Returns}

Ideally we would like to be able to cover the set $\Delta_{n}=\Delta_{0} \backslash\{R<n\}$ with disjoint hyperbolic preballs corresponding to some controlled sequence of hyperbolic times. However, we do not have enough information to carry out such a strategy and there are some technical issues as well. First of all we need to avoid points which are too close to the boundary of $\Delta_{n}$. We write $\Delta_{n}=A_{n} \cup B_{n}$, where $B_{n}$ is a small neighbourhood of $\Delta_{0} \backslash \Delta_{n}$ in $\Delta_{n}$, a kind of buffer zone to smooth out the complicated geometry given by the history of previous returns. In particular, the definition of $B_{n}$ essentially guarantees that any hyperbolic preball $V_{n}(x)$ for $x \in A_{n}$ is completely contained in $\Delta_{n}$. We let $H_{j}$ denote the set of points in $\Delta_{0}$ for which $j$ is a hyperbolic time.

Lemma 5. $\exists c>0$ such that $\forall n \geq 1$ we have $\left|\bigcup_{i=0}^{N}\{x: R(x)=n+i\}\right| \geq$ $c_{0}\left|A_{n-1} \cap H_{n}\right|$.

This says that the proportion of $A_{n-1}$ which has return time between $n$ and $n+N$ is uniformly comparable to the proportion of points in $A_{n}$ for which $n$ is a hyperbolic time. From this we get

Lemma 6. $\exists b>0$ such that $\left|\Delta_{n+N}\right| \leq\left|\Delta_{n}\right| e^{-b \sum_{j=1}^{n}\left|A_{j-1} \cap H_{j}\right| /\left|A_{j-1}\right|}$.

Thus the rate of decay of the $\left|\Delta_{n}\right|$, which is precisely the rate of decay of the tail of the return times, depends on the proportion of each $A_{j-1}$ which has a hyperbolic time at time $j$. In the uniformly expanding case every iterate $j$ is a hyperbolic time for every $x$, and therefore $\left|A_{j-1} \cap H_{j}\right| /\left|A_{j-1}\right| \equiv 1$, giving an exponential decay of the tail of the return times as expected. In our case we can only get that for all $n \geq 1$ and $A \subset M \backslash \Gamma_{n}$, we have $\sum_{j=1}^{n}\left|A \cap H_{j}\right| /|A| \geq \theta n$, as a corollary of Lemma 4

\section{Conclusions}

Thus, intuitively, if the complement of $\Gamma_{n}$ has many hyperbolic times, good returns occur exponentially fast. If $\left|\Gamma_{n}\right|$ decays slowly, then there will come a time that most points which have not yet returned belong to $\Gamma_{n}$, implying that the exponential rate of returns cannot continue until $\left|\Gamma_{n}\right|$ has become sufficiently small again. Thus $\Gamma_{n}$ is a bottleneck slowing down the return times. This idea cannot be completely implemented in practice however, mostly because of the difference between the terms $\sum_{j=1}^{n}\left|A \cap H_{j}\right| /|A|$, which appear in Lemma 4, and the terms $\sum_{j=1}^{n}\left|A_{j-1} \cap H_{j}\right| /\left|A_{j-1}\right|$, which appear in Lemma 6. Given the abstract nature of our assumptions and the definitions of the sets $A_{j}$ and $B_{j}$, the $A_{j}$ may vary much more irregularly than may be expected at first sight. For example, it is possible to envisage a situation in which $\bigcap_{j=1}^{n} A_{j}=\emptyset$. This means that it is not possible to apply the conclusions of Lemma 4 directly to conclude that returns are occurring exponentially fast outside $\Gamma_{n}$. By considering various possible cases, it is nevertheless possible to obtain some good bounds in the case in which $\Gamma_{n}$ is decaying polynomially fast, as stated in the theorem. 


\section{REFERENCES}

[1] J. F. Alves, SRB measures for non-hyperbolic systems with multidimensional expansion, Ann. Scient. Éc. Norm. Sup., $4^{e}$ série, 33 (2000), 1-32. MR 2002i:37032

[2] J. F. Alves, C. Bonatti, M. Viana, SRB measures for partially hyperbolic systems whose central direction is mostly expanding, Invent. Math. 140 (2000), 351-398. MR 2001j:37063a

[3] J. F. Alves, S. Luzzatto, V. Pinheiro, Lyapunov exponent and rates of mixing for onedimensional maps, Preprint 2002.

[4] J. F. Alves, S. Luzzatto, V. Pinheiro, Markov structures and decay of correlations for nonuniformly expanding dynamical systems, Preprint 2002.

[5] J. F. Alves, M. Viana, Statistical stability for robust classes of maps with non-uniform expansion, Ergod. Th. \& Dynam. Sys. 22 (2002), 1-32.

[6] M. Viana, Multidimensional non-hyperbolic attractors, Publ. Math. IHES 85 (1997), 63-96. MR 98f:58146

[7] L.-S. Young, Statistical properties of dynamical systems with some hyperbolicity, Ann. Math. 147 (1998), 585-650. MR 99h:58140

[8] L.-S. Young, Recurrence times and rates of mixing, Israel J. Math. 110 (1999), 153-188. MR 2001j:37062

Departamento de Matemática Pura, Faculdade de Ciências do Porto, Rua do Campo Alegre 687, 4169-007 Porto, Portugal

E-mail address: jfalves@fc.up.pt

$U R L:$ http://www.fc.up.pt/cmup/home/jfalves

Mathematics Department, Imperial College, 180 Queen's Gate, London SW7, UK

E-mail address: stefano.luzzatto@ic.ac.uk

$U R L:$ http://www.ma.ic.ac.uk/ luzzatto

Departamento de Matemática, Universidade Federal da Bahia, Av. Ademar de BarROS S/N, 40170-110 SALVAdor, BraZIL

E-mail address: viltonj@ufba.br 